\title{
Linear Control of Fractional-Order Financial Chaotic Systems with Input Saturation
}

\author{
Junhai Luo, ${ }^{1}$ Guanjun $\mathrm{Li}^{2}$, and Heng Liu ${ }^{2}$ \\ ${ }^{1}$ School of Electronic Engineering, University of Electronic Science and Technology of China, Chengdu 611731, China \\ ${ }^{2}$ Department of Mathematics and Computational Sciences, Huainan Normal University, Huainan 232038, China \\ Correspondence should be addressed to Junhai Luo; junhai_luo@uestc.edu.cn
}

Received 12 March 2014; Accepted 17 July 2014; Published 6 August 2014

Academic Editor: Taishan Yi

Copyright (c) 2014 Junhai Luo et al. This is an open access article distributed under the Creative Commons Attribution License, which permits unrestricted use, distribution, and reproduction in any medium, provided the original work is properly cited.

\begin{abstract}
In this paper, control of fractional-order financial chaotic systems with saturated control input is investigated by means of statefeedback control method. The saturation problem is tackled by using Gronwall-Bellman lemma and a memoryless nonlinearity function. Based on Gronwall inequality and Laplace transform technique, two sufficient conditions are achieved for the asymptotical stability of the fractional-order financial chaotic systems with fractional orders $0<\alpha \leq 1$ and $1<\alpha<2$, respectively. Finally, simulation studies are carried out to show the effectiveness of the proposed linear control method.
\end{abstract}

\section{Introduction}

In the past two decades, studies of chaotic systems have received more and more attention in various fields of natural sciences. This is because chaotic systems are rich in dynamics and possess great sensitivity to initial conditions. Up to now, econophysics has been raised to an alternative scientific methodology to comprehend the highly complex dynamics in economic and financial systems. Many economists are working hard to explain the central features of economic data, including erratic macroeconomic fluctuations (business cycles), irregular microeconomic fluctuations, irregular growth, structural changes, and overlapping waves of economic development $[1,2]$. Representative effects, that is, treated as random shocks, are political events, weather variables, and other human factors [3-7]. Compared with the opinion discussed above, chaos supports an endogenous explanation of the complexity appeared in economic series.

Since chaos in financial systems was firstly studied in 1985 , great impact has been put on the prominent economics recently, because the occurrence of the chaotic phenomenon in the economic system indicates that the macroeconomic operation has in itself the inherent indefiniteness. Studies on the complicated financial systems by using nonlinear method are fruitful $[2,8,9]$. Controlling chaos in fractional-order financial systems is also studied in recent years [10-18]. In [15], an active sliding mode controller is constructed to synchronize fractional-order financial chaotic systems in masterslave structure. In [16], a necessary condition is introduced to confirm the existence of 1-scroll, 2-scroll, or multiscroll chaotic attractors in a fractional-order financial system and a sliding mode controller is proposed. Active control method is also used in [17], and the variable-order fractional derivative is defined in Caputo type. Wang et al. investigate impulsive synchronization and adaptive-impulsive synchronization of a novel financial hyperchaotic system [18]. In above literatures, the stability analysis is carried out based on fractional-order linear system stability theorem and only the situation where fractional order $0<\alpha \leq 1$ is concerned.

Most of real world technical systems are subjected to input constraints, especially in financial systems. In financial systems, input saturation does exist due to a limited size of weather variables, political events, and other human factors. The existence of input saturation may decrease the control performance or cause oscillations and even lead to instability of the system [19-21]. It is advisable for us to consider the control of financial systems with input saturation. For the integer-order linear and nonlinear systems, input saturation 
has received much attention from researchers in the past decade. The sector bounded condition associated with input nonlinearities is useful for analysis and synthesis of control systems subject to input saturation. Then the stability of the system can be formulated using Lyapunov stability theory and invariant theory.

Though many research efforts have been put to the fractional-order financial chaotic systems, the financial systems with saturated control input have rarely been investigated in literatures. Here, with the help of Laplace transform, Mittag-Leffler function, and Gronwall inequality, a linear controller will be derived for fractional-order financial chaotic systems in this paper. There are some main contributions that are worth to be emphasized as follows.

(1) Two sufficient conditions are derived for the asymptotical stability of fractional-order financial chaotic systems with fractional orders $0<\alpha \leq 1$ and $1<$ $\alpha \leq 2$, respectively.

(2) A linear controller is given to control the fractionalorder financial chaotic system.

(3) A memoryless nonlinearity function is employed to handle the input saturation problem in fractionalorder chaotic systems.

\section{Preliminaries and System Description}

2.1. Preliminaries. The Caputo definition of fractional-order derivatives can be expressed as [21-24]

$$
{ }_{0}^{C} D_{t}^{\alpha} x(t)=\frac{1}{\Gamma(n-\alpha)} \int_{0}^{t} \frac{x^{(n)}(\tau)}{(t-\tau)^{\alpha-n+1}} d \tau, \quad n-1<\alpha<n,
$$

where $\alpha$ represents the fractional order and the Euler function $\Gamma(\cdot)$ is defined as $\Gamma(\tau)=\int_{0}^{\infty} t^{\tau-1} e^{-t} d \tau$.

The Laplace transform of Caputo fractional derivative can be given as

$$
\int_{0}^{\infty} e_{0}^{-s t} D_{t}^{\alpha} x(t) d t=s^{\alpha}-\sum_{k=0}^{n-1} s^{\alpha-k-1} x^{(k)}(0) .
$$

The following definition and lemmas will be used.

Definition 1. The Mittag-Leffler function with two parameters can be written as

$$
E_{\alpha, \beta}(z)=\sum_{k=0}^{\infty} \frac{z^{k}}{\Gamma(\alpha k+\beta)}
$$

where $\alpha, \beta>0$ and $z \in C$, and its Laplace transform can be given as

$$
\mathscr{L}\left\{t^{\beta-1} E_{\alpha, \beta}\left(-\lambda t^{\alpha}\right)\right\}=\frac{s^{\alpha-\beta}}{s^{\alpha}+\lambda} .
$$

Lemma 2 (see [22]). If $A \in R^{n \times n}, 0<\alpha \leq 1, \beta$ is an arbitrary real number, and $C>0$ is a real constant, then

$$
E_{\alpha, \beta}(A) \leq \frac{C}{1+\|A\|},
$$

where $\mu \leq|\arg (\operatorname{eig}(A))| \leq \pi$ with $\mu \in R$ satisfying $\pi \alpha / 2<$ $\mu<\min \{\pi, \pi \alpha\}$.

Lemma 3 (see $[24])$. If $t \in[0, T]$ and

$$
x(t) \leq h(t)+\int_{0}^{t} k(\tau) x(\tau) d \tau
$$

where $k(t) \geq 0$ and all the functions involved are continuous on the interval $[0, T]$, then we can obtain

$$
x(t) \leq h(t)+\int_{0}^{t} k(\tau) h(\tau) \exp \left[\int_{\tau}^{t} k(u) d u\right] d \tau .
$$

Definition 4 (see [18]). A memoryless nonlinearity $\varphi(t, x)$ : $[0, \infty) \times R^{p} \rightarrow R^{p}$ is said to satisfy a sector condition if the following inequality holds:

$$
\left(\varphi(t, x)-K_{1} x\right)^{T}\left(\varphi(t, x)-K_{2} x\right) \leq 0, \quad \forall x \in S
$$

for constant matrices $K_{1}$ and $K_{2}$, where $K_{2}-K_{1}$ is a symmetric positive matrix and $S$ contains the origin.

Based on the Definition 4, the following lemma holds.

Lemma 5 (see [18]). Let

$$
S\left(L K, u_{0}\right)=\left\{x(t) \in R^{n} \mid-u_{0} \leq L K x(t) \leq u_{0}\right\},
$$

where $L=\operatorname{diag}\left[l_{1}, l_{2}, \ldots, l_{n}\right]$ with $0<l_{i} \leq 1, \forall i=1,2, \ldots, n$, and

$$
\varphi(t, x(t))=\operatorname{sat}(K x(t))-L K x(t) ;
$$

then the following inequalities are equivalent:
(1) $(\operatorname{sat}(K x(t))-L K x(t))^{T}(\operatorname{sat}(K x(t))-K x(t)) \leq 0$;
(2) $\varphi(t, x(t))^{T}(\varphi(t, x(t))-(K-L K) x(t)) \leq 0$;
(3) $\|\varphi(t, x(t))\| \leq\|K-L K\|\|x(t)\|$.

Lemma 6. The autonomous dynamic system

$$
D^{\alpha} x(t)=A x(t), \quad x(0)=x_{0}
$$

is asymptotically stable if the following condition holds:

$$
|\arg (\operatorname{eig}(A))|>\frac{\pi \alpha}{2} \text {. }
$$

The stability region for $0<\alpha<1$ is depicted in Figure 1 .

2.2. Description of Fractional-Order Financial Chaotic Systems. The fractional-order financial chaotic systems are proposed by [1]. The mathematical model describes a fractionalorder financial system including three nonlinear differential equations. The states, $x_{1}(t), x_{2}(t)$, and $x_{3}(t)$, represent the interest rate, the investment demand, and the price index, respectively. The fractional-order model of the system can be described as

$$
\begin{gathered}
{ }_{0}^{C} D_{t}^{\alpha} x_{1}(t)=x_{3}(t)+\left(x_{2}(t)-a\right) x_{1}(t), \\
{ }_{0}^{C} D_{t}^{\alpha} x_{2}(t)=1-b x_{2}(t)-x_{1}^{2}(t), \\
{ }_{0}^{C} D_{t}^{\alpha} x_{3}(t)=-x_{1}(t)-c x_{3}(t),
\end{gathered}
$$




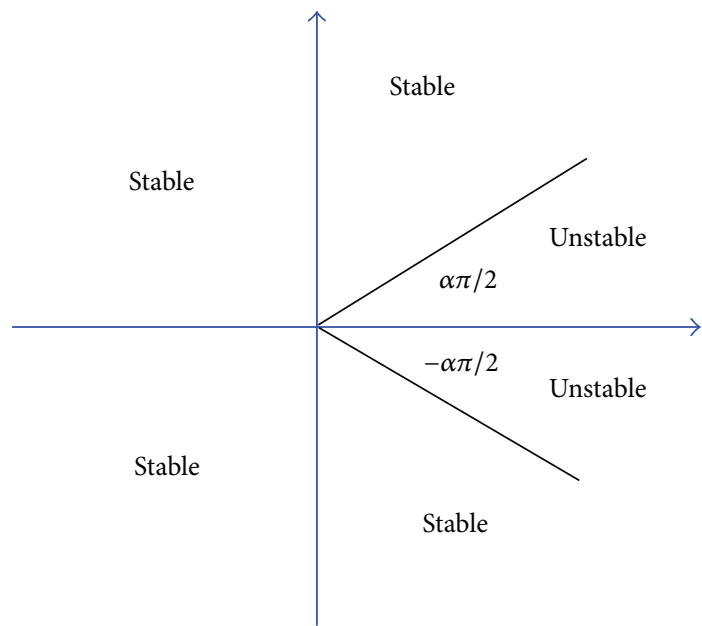

FIGURE 1: Stability region of linear system (11) with fractional order $0<\alpha<1$.

where $a$ denotes the saving amount, $b$ is the cost per investment, and $c$ is the elasticity of demand of commercial market. $0<\alpha<2$ is the fractional-order derivative.

\section{State-Feedback Controller Design and Stability Analysis}

3.1. Fractional Order $\alpha$ : $0<\alpha \leq 1$. Let us rewrite the controlled system (13) as the following compact form:

$$
\begin{array}{r}
{ }_{0}^{C} D_{t}^{\alpha} x(t)=P x(t)+f(x(t))+[0,1,0]^{T}+\operatorname{sat}(u(t)), \\
x(0)=x_{0},
\end{array}
$$

where $x(t), u(t) \in R^{3}$, represent the state variables and the control input, respectively. Consider that

$$
\begin{gathered}
P=\left[\begin{array}{ccc}
-a & 0 & 1 \\
0 & -b & 0 \\
-1 & 0 & -c
\end{array}\right], \\
f(x)=\left[\begin{array}{c}
x_{1}(t) x_{2}(t) \\
-x_{1}^{2}(t) \\
0
\end{array}\right] \\
\operatorname{sat}(u)=\left[\operatorname{sat}\left(u_{1}\right) \text {, sat }\left(u_{2}\right), \operatorname{sat}\left(u_{3}\right)\right]^{T}
\end{gathered}
$$

is the vector-valued saturation function with

$$
\operatorname{sat}\left(u_{i}\right)=\operatorname{sign}\left(u_{i}\right) \min \left(u_{0 i},\left|u_{i}\right|\right), \quad i=1,2,3,
$$

where $u_{0 i}$ represents the symmetric saturation level of the $i$ th control input.

Noting that in chaotic systems the states are bounded, the nonlinear function $f(x)$ satisfies

$$
\|f(x(t))\|=\left|x_{1}(t)\right| \sqrt{x_{1}^{2}(t)+x_{2}^{2}(t)} \leq M\|x(t)\|,
$$

where $M>\left|x_{1}(t)\right|$ is a constant.
Let us define the state-feedback control input as

$$
u(t)=K x(t)+[0,-1,0]^{T},
$$

where $K \in R^{3 \times 3}$ is the control gain matrix. Then we have

$$
\begin{aligned}
{ }_{0}^{C} D_{t}^{\alpha} x(t)= & P x(t)+[0,1,0]^{T}+f(x(t)) \\
& +\operatorname{sat}\left(K x(t)+[0,-1,0]^{T}\right) \\
= & A x(t)+f(x(t))+\varphi(t, x(t)),
\end{aligned}
$$

where $A=P+L K$.

Theorem 7. Consider system (14). If we choose matrices $L$ and $K$ such that $|\arg (\operatorname{eig}(A))|>\alpha \pi / 2$ and $\alpha\|A\|>d$, then system (14) is asymptotically stable. $d$ is a positive constant and will be defined later.

Proof. Taking Laplace transform on (19), we can obtain

$$
s^{\alpha} X(s)=s^{\alpha-1} x_{0}+A X(s)+\mathscr{L}\{f(x)\}+\mathscr{L}\{\varphi(t, x)\},
$$

where $X(s)$ represents the Laplace transform of $x(t)$. Let $I$ denote the $3 \times 3$ identity matrix; we have

$$
X(s)=\left(I s^{\alpha}-A\right)^{-1}\left(s^{\alpha-1} x_{0}+\mathscr{L}\{f(x)\}+\mathscr{L}\{\varphi(t, x)\}\right) .
$$

By taking Laplace inverse transform on (21), we get the solution of system (14):

$$
\begin{aligned}
x(t)=E_{q, 1}\left(A(t)^{\alpha}\right) x_{0}+\int_{0}^{t}(t-\tau)^{\alpha-1} & \\
& \times E_{\alpha, \alpha}\left(A(t-\tau)^{\alpha}\right) f(x) d \tau \\
& +\int_{0}^{t}(t-\tau)^{\alpha-1} E_{\alpha, \alpha}\left(A(t-\tau)^{\alpha}\right) \varphi(t, x) d \tau .
\end{aligned}
$$

According to Lemma 2, we know there exist some constants $c_{i}>0, i=1,2$, such that

$$
\begin{aligned}
\|x(t)\| \leq & \frac{c_{1}\left\|x_{0}\right\|}{1+\|A\| t^{\alpha}}+\int_{0}^{t} \frac{(t-\tau)^{\alpha-1} c_{2}}{1+\|A\|(t-\tau)^{\alpha}}\|f(x)\| d \tau \\
& +\int_{0}^{t} \frac{(t-\tau)^{\alpha-1} \mathcal{c}_{2}}{1+\|A\|(t-\tau)^{\alpha}}\|\varphi(t, x)\| d \tau .
\end{aligned}
$$

Then (23) can be rewritten as

$$
\begin{aligned}
\|x(t)\| \leq & \frac{c_{1}\left\|x_{0}\right\|}{1+\|A\| t^{\alpha}}+M \int_{0}^{t} \frac{(t-\tau)^{\alpha-1} c_{2}}{1+\|A\|(t-\tau)^{\alpha}}\|x(t)\| d \tau \\
& +\int_{0}^{t} \frac{(t-\tau)^{\alpha-1} c_{2}}{1+\|A\|(t-\tau)^{\alpha}}\|\varphi(t, x)\| d \tau .
\end{aligned}
$$

From Definition 4 and Lemma 5, we know that $\varphi(t, x(t))$ satisfies

$$
\|\varphi(t, x(t))\| \leq\|K-L K\|\|x(t)\| .
$$


Let $d=M c_{2}+c_{2}\|K-L K\|$; we can obtain

$\|x(t)\| \leq \frac{c_{1}\left\|x_{0}\right\|}{1+\|A\| t^{\alpha}}+d \int_{0}^{t} \frac{(t-\tau)^{\alpha-1}}{1+\|A\|(t-\tau)^{\alpha}}\|x(t)\| d \tau$.

By using Lemma 3, we have

$$
\begin{aligned}
\|x(t)\| \leq & \frac{c_{1}\left\|x_{0}\right\|}{1+\|A\| t^{\alpha}} \\
& +\int_{0}^{t} \frac{d c_{1}(t-\tau)^{\alpha-1}\left\|x_{0}\right\|}{\left(1+\|A\|(t-\tau)^{\alpha}\right)\left(1+\|A\| \tau^{\alpha}\right)} \\
& \times \exp \left(\int_{\tau}^{t} \frac{d(t-y)^{\alpha-1}}{1+\|A\|(t-y)^{\alpha}} d y\right) d \tau \\
= & \frac{c_{1}\left\|x_{0}\right\|}{1+\|A\| t^{\alpha}} \\
& +\int_{0}^{t} \frac{d c_{1}(t-\tau)^{\alpha-1}\left\|x_{0}\right\|}{\left(1+\|A\| \tau^{\alpha}\right)\left(1+\|A\|(t-\tau)^{\alpha}\right)^{1-c / \alpha\|A\|}} d \tau \\
\leq & \frac{c_{1}\left\|x_{0}\right\|}{1+\|A\| t^{\alpha}} \\
& +d c_{1}\left\|x_{0}\right\|\|A\|^{d / \alpha\|A\|-2} \int_{0}^{t}(t-\tau)^{d /\|A\|-1} \tau^{-\alpha} d \tau \\
= & \frac{c_{1}\left\|x_{0}\right\|}{1+\|A\| t^{\alpha}}+d c_{1}\left\|x_{0}\right\|\|A\|^{c / \alpha\|A\|-2} \\
& \times \frac{\Gamma(d /\|A\|) \Gamma(1-\alpha)}{\Gamma(1+d /\|A\|-\alpha)} t^{d /\|A\|-\alpha} .
\end{aligned}
$$

Since $\alpha\|A\|>d$, from (27) we can conclude that

$$
\lim _{t \rightarrow \infty}\|x(t)\|=0,
$$

and this ends the proof.

3.2. Fractional Order $\alpha$ : $1<\alpha<2$. Let the initial conditions be $x^{(i)}(0)=x_{i}, i=1,2$. Then we have the following results.

Theorem 8. Consider system (14). If we choose matrices $L$ and $K$ such that $|\arg (\operatorname{eig}(A))|>\alpha \pi / 2$ and $(\alpha-1)\|A\|>d$, then system (14) is asymptotically stable. $d$ is a positive constant.

Proof. Similar to the proof of Theorem 8, taking the Laplace transform and Laplace inverse transform on (14) gives

$$
\begin{aligned}
x(t)= & E_{q, 1}\left(A(t)^{\alpha}\right) x_{1}+\int_{0}^{t}(t-\tau)^{\alpha-1} E_{\alpha, \alpha}\left(A(t-\tau)^{\alpha}\right) f(x) d \tau \\
& +\int_{0}^{t}(t-\tau)^{\alpha-1} E_{\alpha, \alpha}\left(A(t-\tau)^{\alpha}\right) \varphi(t, x) d \tau \\
& +t E_{\alpha, 2}\left(A t^{\alpha}\right) x_{2} .
\end{aligned}
$$

According to Lemma 2, there exist positive constants $c_{i}, i=1,2,3$, such that

$$
\begin{aligned}
\|x(t)\| \leq & \frac{c_{1}\left\|x_{1}\right\|}{1+\|A\| t^{\alpha}}+\int_{0}^{t} \frac{(t-\tau)^{\alpha-1} c_{2}}{1+\|A\|(t-\tau)^{\alpha}}\|f(x)\| d \tau \\
& +\int_{0}^{t} \frac{(t-\tau)^{\alpha-1} c_{2}}{1+\|A\|(t-\tau)^{\alpha}}\|\varphi(t, x)\| d \tau+\frac{c_{3} t\left\|x_{2}\right\|}{1+\|A\| t^{\alpha}} .
\end{aligned}
$$

From Definition 4 and Lemma 5, we know that $\varphi(t, x(t))$ satisfies

$$
\|\varphi(t, x(t))\| \leq\|K-L K\|\|x(t)\| .
$$

Let $d=M c_{2}+c_{2}\|K-L K\|$; we can obtain

$$
\begin{aligned}
\|x(t)\| \leq & \frac{c_{1}\left\|x_{1}\right\|+c_{3} t\left\|x_{1}\right\|}{1+\|A\| t^{\alpha}} \\
& +d \int_{0}^{t} \frac{(t-\tau)^{\alpha-1}}{1+\|A\|(t-\tau)^{\alpha}}\|x(t)\| d \tau .
\end{aligned}
$$

Then we have

$$
\begin{aligned}
& \|x(t)\| \leq \frac{c_{1}\left\|x_{1}\right\|+c_{3} t\left\|x_{1}\right\|}{1+\|A\| t^{\alpha}} \\
& +\int_{0}^{t} \frac{d(t-\tau)^{\alpha-1}\left(c_{1}\left\|x_{1}\right\|+c_{3} t\left\|x_{2}\right\|\right)}{\left(1+\|A\|(t-\tau)^{\alpha}\right)\left(1+\|A\| \tau^{\alpha}\right)} \\
& \times \exp \left(\int_{\tau}^{t} \frac{d(t-y)^{\alpha-1}}{1+\|A\|(t-y)^{\alpha}} d y\right) d \tau \\
& =\frac{c_{1}\left\|x_{1}\right\|+c_{3} t\left\|x_{1}\right\|}{1+\|A\| t^{\alpha}} \\
& +\int_{0}^{t} \frac{d(t-\tau)^{\alpha-1}\left(c_{1}\left\|x_{1}\right\|+c_{3}\left\|x_{2}\right\|\right)}{\left(1+\|A\| \tau^{\alpha}\right)\left(1+\|A\|(t-\tau)^{\alpha}\right)^{1-c / \alpha\|A\|}} d \tau \\
& \leq \frac{c_{1}\left\|x_{1}\right\|+c_{3} t\left\|x_{1}\right\|}{1+\|A\| t^{\alpha}} \\
& +d c_{1}\left\|x_{1}\right\|\|A\|^{d / \alpha\|A\|-2} \int_{0}^{t}(t-\tau)^{1 /\|A\|-1} \tau^{-\alpha} d \tau \\
& +d c_{3}\left\|x_{2}\right\|\|A\|^{d / \alpha\|A\|-2} \int_{0}^{t}(t-\tau)^{1 /\|A\|-1} \tau^{1-\alpha} d \tau \\
& =\frac{c_{1}\left\|x_{1}\right\|+c_{3} t\left\|x_{1}\right\|}{1+\|A\| t^{\alpha}}+d c_{1}\left\|x_{1}\right\|\|A\|^{d / \alpha\|A\|-2} \\
& \times \frac{\Gamma(d /\|A\|) \Gamma(1-\alpha)}{\Gamma(1+d /\|A\|-\alpha)} t^{d /\|A\|-\alpha} \\
& +d c_{3}\left\|x_{2}\right\|\|A\|^{d / \alpha\|A\|-2} \\
& \times \frac{\Gamma(d /\|A\|) \Gamma(2-\alpha)}{\Gamma(2+d /\|A\|-\alpha)} t^{d /\|A\|+1-\alpha} .
\end{aligned}
$$


Since $(\alpha-1)\|A\|>d$, from (33) we know that

$$
\lim _{t \rightarrow \infty}\|x(t)\|=0
$$

and this ends the proof.

\section{Simulation Studies}

The system (14) has 3 equilibriums:

$$
\begin{gathered}
E_{1}=\left(0 ; \frac{1}{b} ; 0\right), \\
E_{2}=\left(\sqrt{\frac{c-b-a b c}{c}} ; \frac{1+a c}{c} ;-\frac{1}{c} \sqrt{\frac{c-b-a b c}{c}}\right), \\
E_{3}=\left(-\sqrt{\frac{c-b-a b c}{c}} ; \frac{1+a c}{c} ; \frac{1}{c} \sqrt{\frac{c-b-a b c}{c}}\right) .
\end{gathered}
$$

The Jacobian matrix of the fractional-order chaotic system (14), at the equilibrium $E^{*}=\left[x^{*}, y^{*}, z^{*}\right]^{T}$, can be given as

$$
J_{E}=\left[\begin{array}{ccc}
-a+y^{*} & x^{*} & 1 \\
-2 x^{*} & -b & 0 \\
-1 & 0 & -c
\end{array}\right]
$$

Let $a=1, b=0.1$, and $c=1$. The eigenvalues for the system equilibrium $E_{1}=(0 ; 10 ; 0)$ are $\lambda_{1}=8.8990, \lambda_{2}=$ -0.8990 , and $\lambda_{3}=-0.1$. And it is a saddle point. For equilibrium points $E_{2}=(0.8944 ; 2 ;-0.8944)$ and $E_{3}=$ $(-0.8944 ; 2 ; 0.8944)$ they are $\lambda_{1}=-0.7609$ and $\lambda_{2,3}=$ $0.3304 \pm 1.4112 i$. It is a saddle-focus point. Since it is an unstable equilibrium, the condition for chaos is satisfied and the system (14) can show chaotic behavior. We can easily get the minimal commensurate order of the system which is $\alpha>0.8537$.

Case $1(0<\alpha \leq 1)$. Assume the fractional order is $\alpha=0.9$. The characteristic equation of the linearized system for the equilibrium $E_{1}$ is

$$
\lambda^{27}-7.9 \lambda^{18}-8.8 \lambda^{9}-0.8=0 .
$$

The characteristic equation of the linearized system for the equilibriums $E_{2}$ and $E_{3}$ is

$$
\lambda^{27}+0.1 \lambda^{18}+1.6 \lambda^{9}+1.6=0
$$

and the unstable eigenvalues are $\lambda_{1,2}=1.0306 \pm 0.1547 i$.

Let the initial condition be $x(0)=[2,-1,1]^{T}$. The chaotic behavior of uncontrolled fractional-order financial system (13) is shown in Figure 2.

The control gain matrices are chosen as $L=$ $\operatorname{diag}[0.9,0.9,0.9]$.

Let

$$
A=\left[\begin{array}{ccc}
0 & 1 & 0 \\
0 & 0 & 1 \\
-2 & -2 & -3
\end{array}\right]
$$

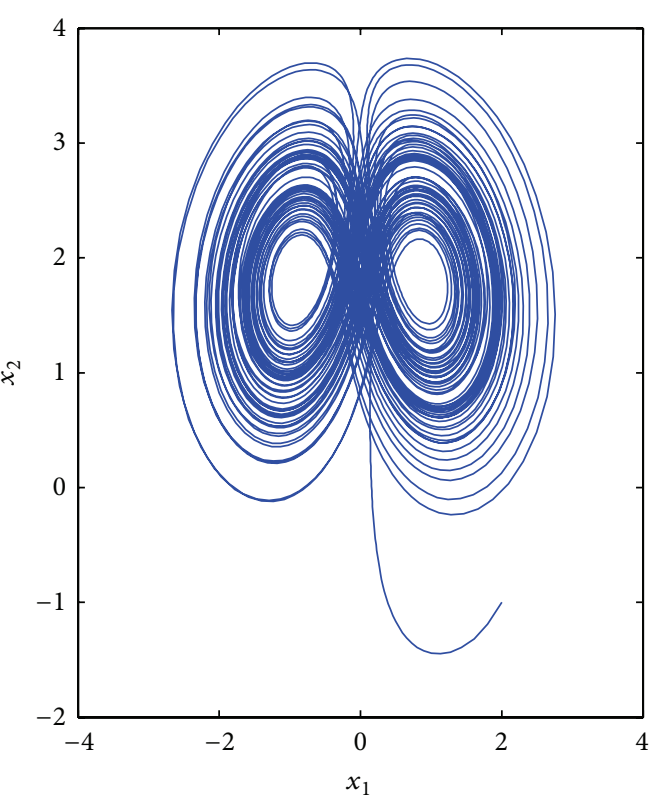

(a)

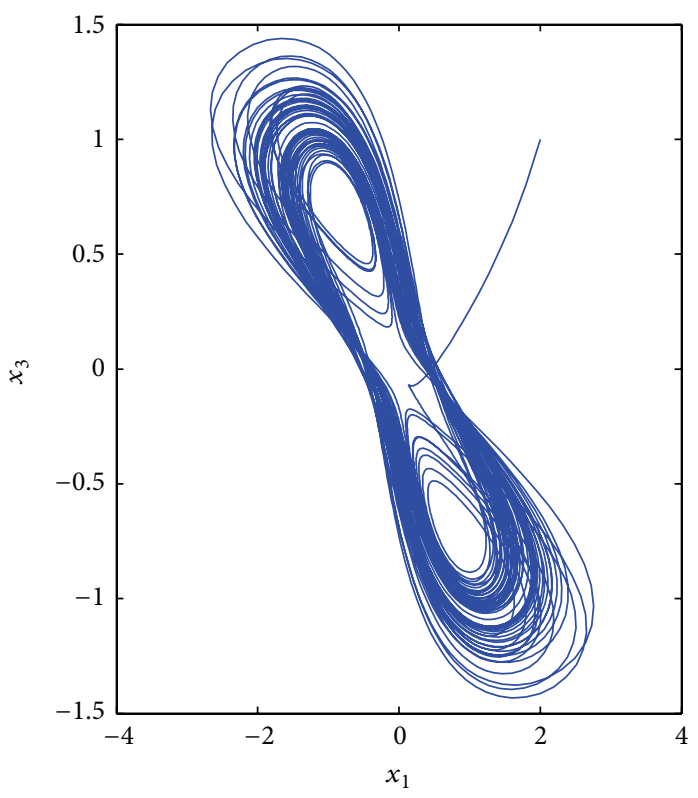

(b)

FIGURE 2: Chaotic attractor of fractional-order financial system with fractional order $\alpha=0.90$ : (a) $x_{1}-x_{2}$ plane; (b) $x_{1}-x_{3}$ plane.

then we have $\|A\|=4.2160$. From simulation results (see Figure 2), we know $\left|x_{1}(t)\right| \leq 3$. From $A=P+L K$, we have

$$
K=\left[\begin{array}{ccc}
1.1111 & 1.1111 & -1.1111 \\
0 & 0.1111 & 1.1111 \\
-1.1111 & -2.2222 & -2.2222
\end{array}\right]
$$

From above discussion, we know $\|A\|=4.2160$ and $|\arg (\operatorname{eig}(A))|=[3.1416,1.8428,1.8428]^{T}$. Then we can easily test that the conditions $|\arg (\operatorname{eig}(A))|>\alpha \pi / 2$ and $\alpha\|A\|>d$ are satisfied. 


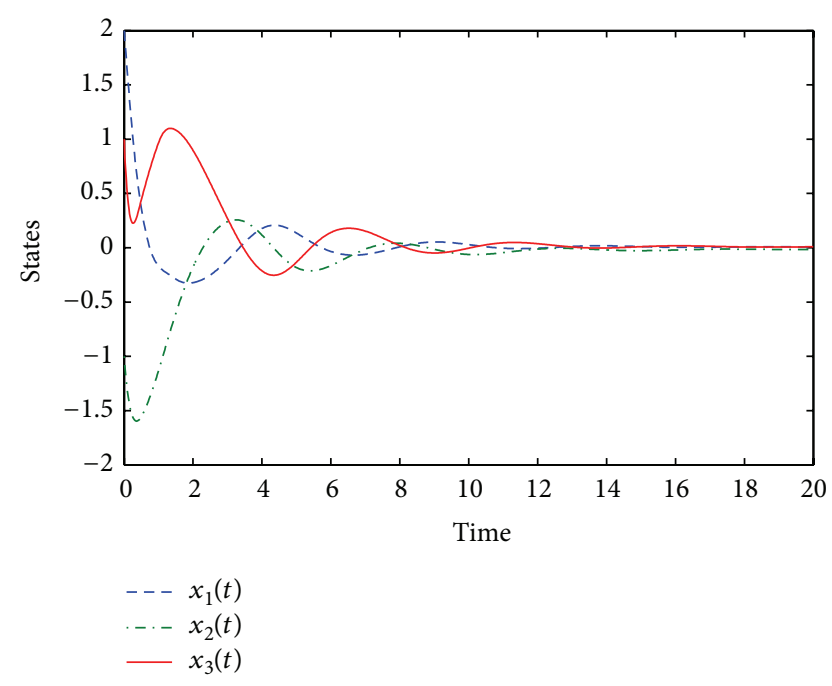

FIGURE 3: Time response of the state variables with fractional order $\alpha=0.90$.

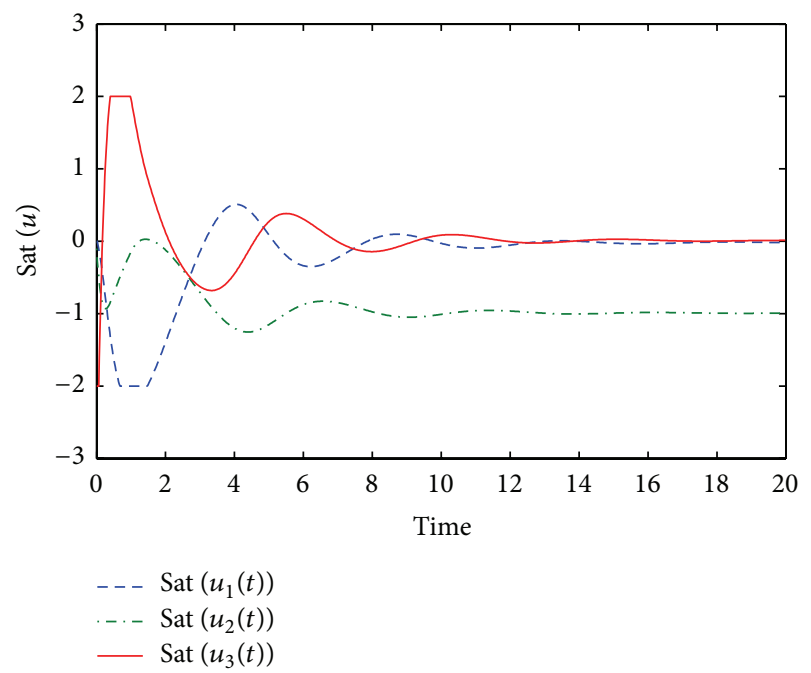

FIGURE 4: Time response of the control inputs with fractional order $\alpha=0.90$.

Let $u_{01}=u_{02}=u_{03}=2$. The simulation results can be seen in Figures 3 and 4. From the results, we can see that the states variables converge rapidly. The involved system is asymptotic stable. Figure 4 shows the boundedness and smoothness of the saturated control inputs. It can be concluded that good control performance has been achieved.

Case $2(1<\alpha<2)$. Let the fractional order be $\alpha=1.04$. The chaotic behavior is depicted in Figure 5. In the simulation, the control gain matrices are chosen as $L=\operatorname{diag}[0.9,0.9,0.9]$,

$$
K=\left[\begin{array}{ccc}
1.1111 & 1.1111 & -1.1111 \\
0 & 0.1111 & 1.1111 \\
-4.4444 & -6.6667 & -6.6667
\end{array}\right]
$$

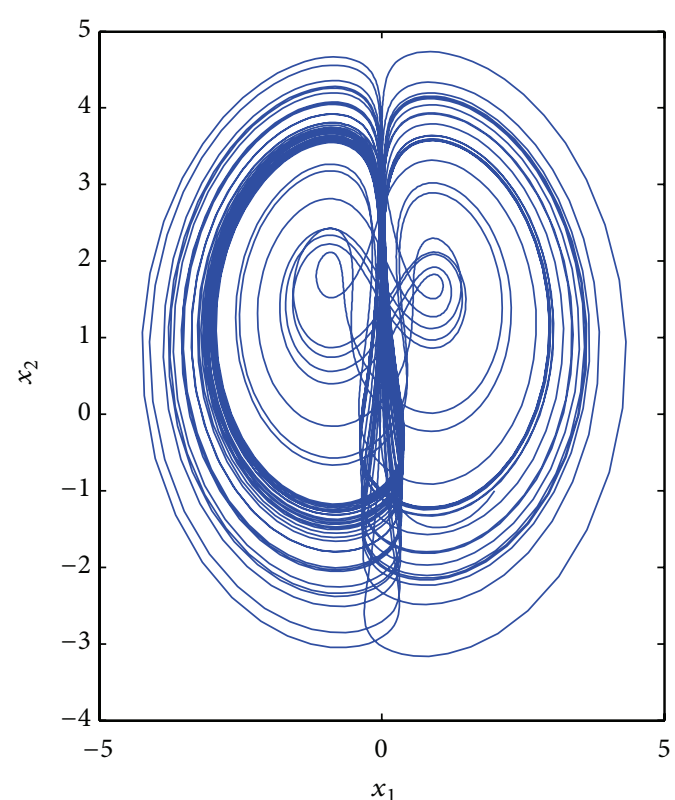

(a)

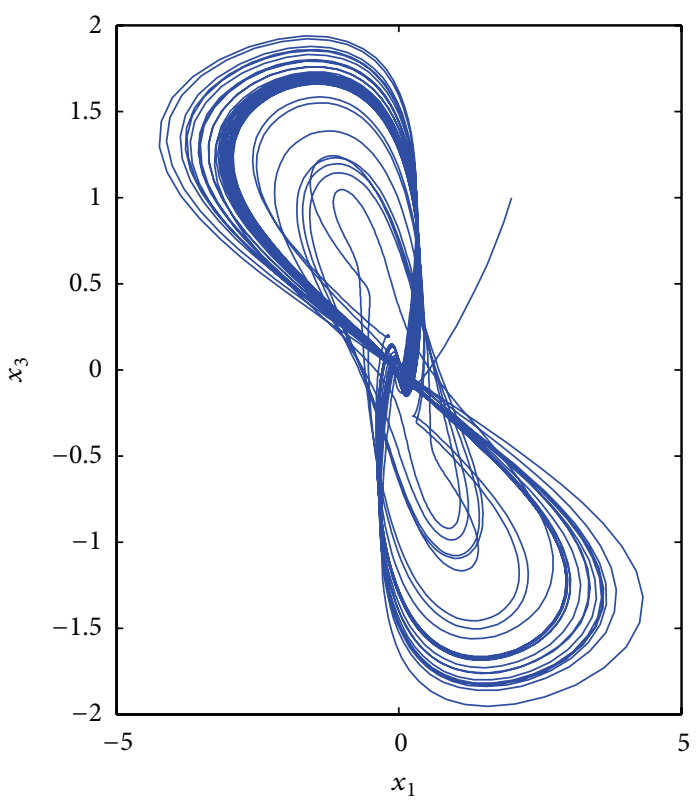

(b)

FIGURE 5: Chaotic attractor of fractional-order financial system with fractional order $\alpha=1.04$ : (a) $x_{1}-x_{2}$ plane; (b) $x_{1}-x_{3}$ plane.

then we have

$$
A=P+L K=\left[\begin{array}{ccc}
0 & 1 & 0 \\
0 & 0 & 1 \\
-5 & -6 & -7
\end{array}\right] .
$$

From above discussion, we know $\|A\|=10.5249$ and $|\arg (\operatorname{eig}(A))|=[3.1416,2.0573,2.0573]^{T}$. Then we have $|\arg (\operatorname{eig}(A))|>\alpha \pi / 2$ and $\alpha\|A\|>d$.

Let $u_{01}=u_{02}=u_{03}=2$. The simulation results can be seen in Figures 6 and 7. From the simulation results we can conclude that good control performance has been achieved. 


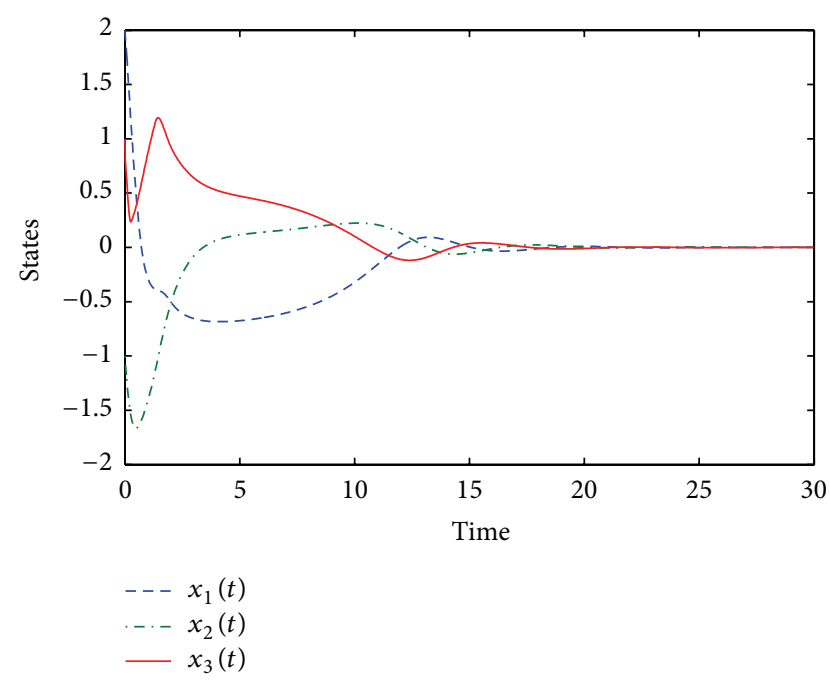

Figure 6: Time response of the state variables with fractional order $\alpha=1.04$.

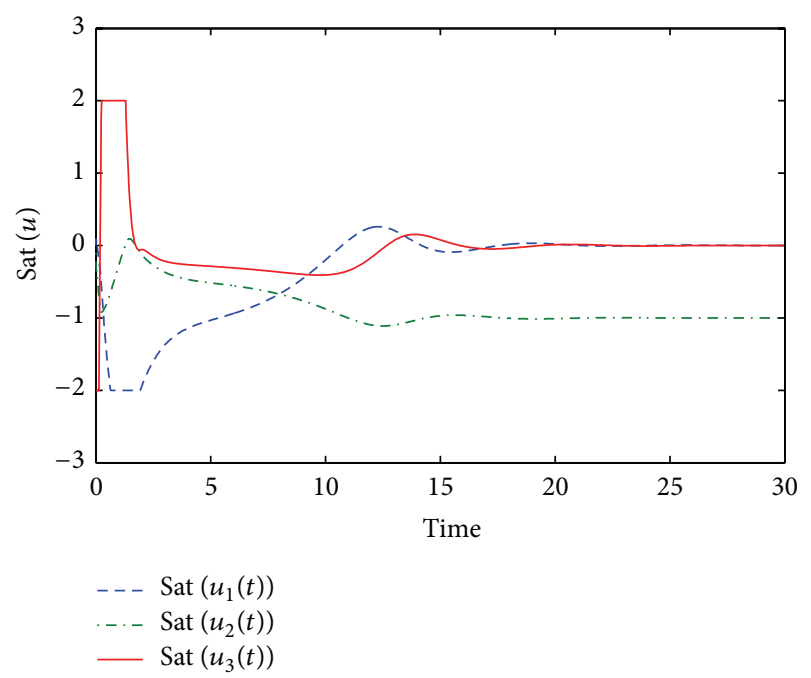

FIGURE 7: Time response of the control inputs with fractional order $\alpha=1.04$.

\section{Conclusions}

We investigate the control problem for fractional-order financial chaotic systems subject to input saturation by means of linear control. Two sufficient conditions are given for the stabilization of such systems with fractional orders $0<\alpha \leq 1$ and $1<\alpha<2$, respectively. A state-feedback controller is designed and the asymptotical stability of the involved system is guaranteed. It is shown that state-feedback controller can be designed to control the fractional-order financial chaotic systems. Simulation studies confirm the results of this paper.

\section{Conflict of Interests}

The authors do not have a direct financial relation with any commercial identity mentioned in their paper that might lead to a conflict of interests for any of the authors.

\section{Acknowledgments}

This work is supported by the National Natural Science Foundation of China (Grant no. 61001086) and the Fundamental Research Funds for the Central Universities (Grant no. ZYGX2011X004).

\section{References}

[1] W. C. Chen, "Nonlinear dynamics and chaos in a fractionalorder financial system," Chaos, Solitons and Fractals, vol. 36, no. 5, pp. 1305-1314, 2008.

[2] G. Cai and J. Huang, "A new finance chaotic attractor," International Journal of Nonlinear Science, vol. 3, no. 3, pp. 213-220, 2007.

[3] F. Wen and X. Yang, "Skewness of return distribution and coefficient of risk premium," Journal of Systems Science \& Complexity, vol. 22, no. 3, pp. 360-371, 2009.

[4] C. Huang, C. Peng, X. Chen, and F. Wen, "Dynamics analysis of a class of delayed economic model," Abstract and Applied Analysis, vol. 2013, Article ID 962738, 12 pages, 2013.

[5] C. Huang, X. Gong, X. Chen, and F. Wen, "Measuring and forecasting volatility in Chinese stock market using HAR-CJM model," Abstract and Applied Analysis, vol. 2013, Article ID 143194, 13 pages, 2013.

[6] F. Wen and Z. Liu, "A copula-based correlation measure and its application in chinese stock market," International Journal of Information Technology and Decision Making, vol. 8, no. 4, pp. 787-801, 2009.

[7] F. Wen, X. Gong, Y. Chao, and X. Chen, "The effects of prior outcomes on risky choice: evidence from the stock market," Mathematical Problems in Engineering, vol. 2014, Article ID 272518, 8 pages, 2014.

[8] M. Rivero, J. J. Trujillo, L. Vázquez, and M. P. Velasco, "Fractional dynamics of populations," Applied Mathematics and Computation, vol. 218, no. 3, pp. 1089-1095, 2011.

[9] Z. Wang, X. Huang, and H. Shen, "Control of an uncertain fractional order economic system via adaptive sliding mode," Neurocomputing, vol. 83, pp. 83-88, 2012.

[10] J. Cao, D. W. C. Ho, and Y. Yang, "Projective synchronization of a class of delayed chaotic systems via impulsive control," Physics Letters. A, vol. 373, no. 35, pp. 3128-3133, 2009.

[11] J. D. Cao and L. L. Li, "Cluster synchronization in an array of hybrid coupled neural networks with delay," Neural Networks, vol. 22, no. 4, pp. 335-342, 2009.

[12] J. Cao, A. Alofi, A. Al-Mazrooei, and A. Elaiw, "Synchronization of switched interval networks and applications to chaotic neural networks," Abstract and Applied Analysis, vol. 2013, Article ID 940573, 11 pages, 2013.

[13] H. Zhang, J. Cao, and W. Jiang, "Controllability criteria for linear fractional differential systems with state delay and impulses," Journal of Applied Mathematics, vol. 2013, Article ID 146010, 9 pages, 2013.

[14] J. D. Cao and Y. Wan, "Matrix measure strategies for stability and synchronization of inertial BAM neural network with time delays," Neural Networks, vol. 53, pp. 165-172, 2014.

[15] M. S. Tavazoei and M. Haeri, "Synchronization of chaotic fractional-order systems via active sliding mode controller," Physica A: Statistical Mechanics and Its Applications, vol. 387, no. 1, pp. 57-70, 2008. 
[16] M. S. Tavazoei and M. Haeri, "Chaotic attractors in incommensurate fractional order systems," Physica D: Nonlinear Phenomena, vol. 237, no. 20, pp. 2628-2637, 2008.

[17] Y. F. Xu and Z. M. He, "Synchronization of variable-order fractonal financial systems via active control method," Central European Journal of Physics, vol. 11, no. 6, pp. 824-835, 2013.

[18] Z. Wang, X. Huang, and G. D. Shi, "Analysis of nonlinear dynamics and chaos in a fractional order financial system with time delay," Computers \& Mathematics with Applications, vol. 62, no. 3, pp. 1531-1539, 2011.

[19] Y.-H. Lim, K.-K. Oh, and H.-S. Ahn, "Stability and stabilization of fractional-order linear systems subject to input saturation," IEEE Transactions on Automatic Control, vol. 58, no. 4, pp. 10621067, 2013.

[20] T. Gußner, M. Jost, and J. Adamy, "Controller design for a class of nonlinear systems with input saturation using convex optimization," Systems and Control Letters, vol. 61, no. 1, pp. 258$265,2012$.

[21] J. Luo, "State-feedback control for fractional-order nonlinear systems subject to input saturation," Mathematical Problems in Engineering, vol. 2014, Article ID 891639, 8 pages, 2014.

[22] I. Podlubny, Fractional Differential Equations, vol. 198, Mathematics in Science and Engineering, Academic Press, San Diego, Calif, USA, 1999.

[23] H. S. Ahn and Y. Q. Chen, "Necessary and sufficient stability condition of fractional-order interval linear systems," Automatica, vol. 44, no. 11, pp. 2985-2988, 2008.

[24] L. Chen, Y. Chai, R. Wu, and J. Yang, "Stability and stabilization of a class of nonlinear fractional-order systems with caputo derivative," IEEE Transactions on Circuits and Systems II: Express Briefs, vol. 59, no. 9, pp. 602-606, 2012. 


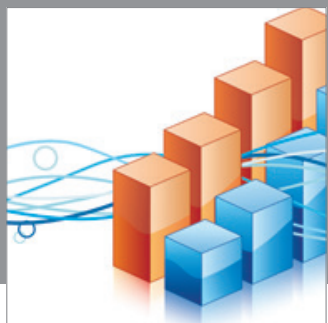

Advances in

Operations Research

mansans

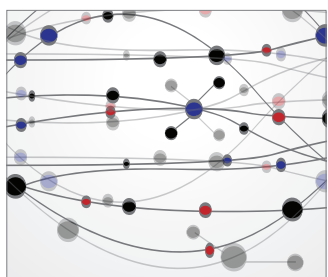

The Scientific World Journal
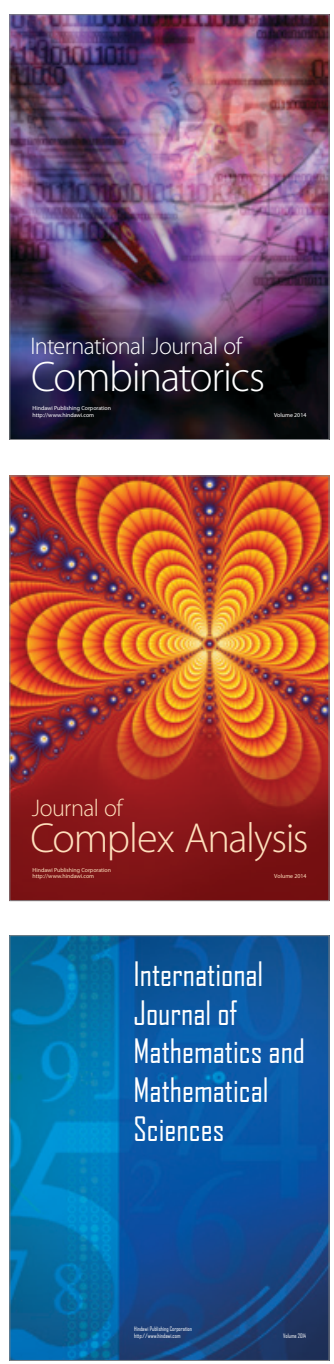
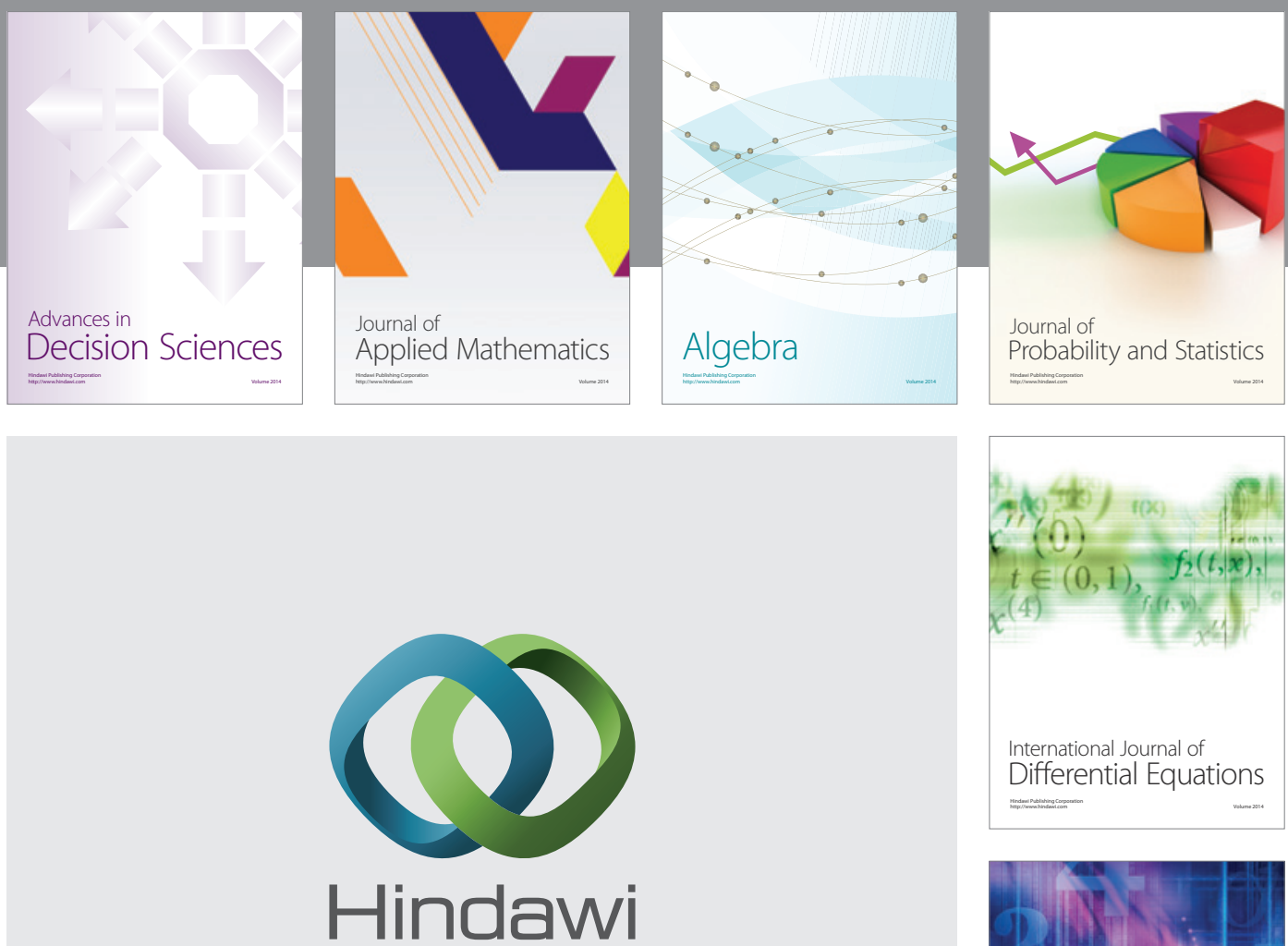

Submit your manuscripts at http://www.hindawi.com
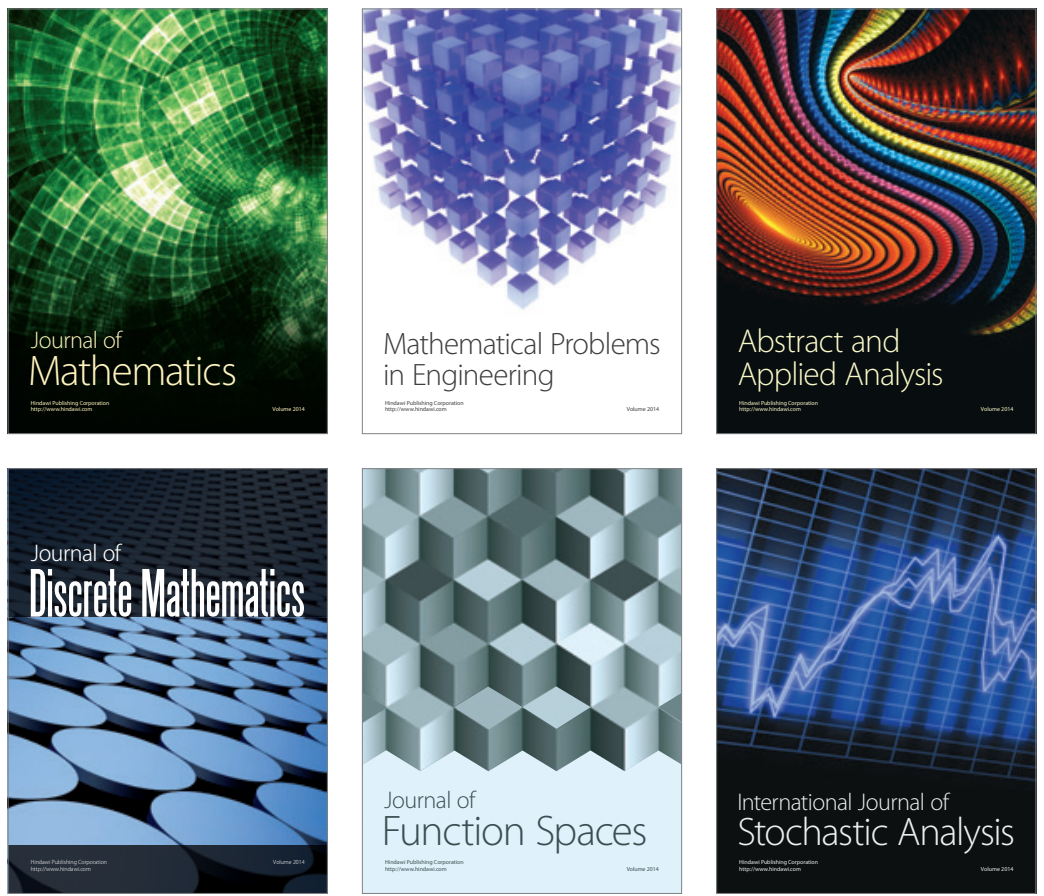

Journal of

Function Spaces

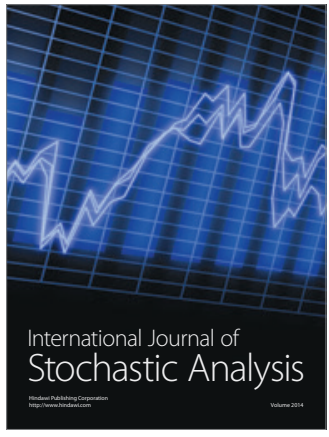

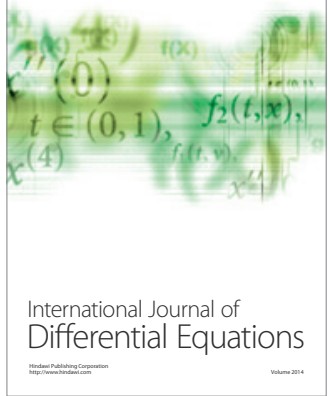
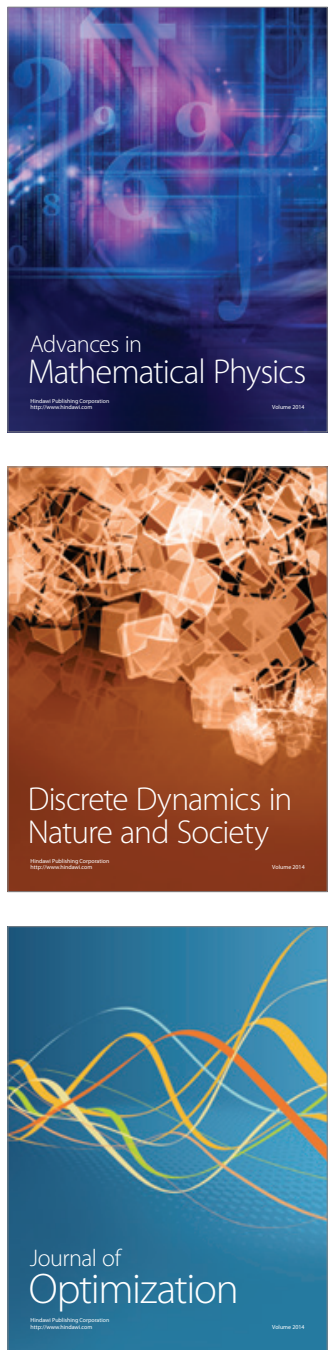